\title{
Temporal changes in serum creatine kinase concentration and degree of muscle rigidity in 24 patients with neuroleptic malignant syndrome
}

This article was published in the following Dove Press journal:

Neuropsychiatric Disease and Treatment

17 June 2013

Number of times this article has been viewed

\author{
Koichi Nisijima \\ Katutoshi Shioda \\ Department of Psychiatry, Jichi \\ Medical University, Tochigi, Japan
}

Correspondence: Koichi Nisijima Department of Psychiatry,

Yakushiji Shimotuke-Shi,

Tochigi-Ken, 329-0498, Japan

$\mathrm{Tel}+8 \mathrm{I} 285587364$

Fax +8I 285446198

Email midorin@jichi.ac.jp

\begin{abstract}
Neuroleptic malignant syndrome (NMS) is a dangerous adverse response to antipsychotic drugs. It is characterized by the four major clinical symptoms of hyperthermia, severe muscle rigidity, autonomic dysfunction, and altered mental state. Serum creatine kinase (CK) elevation occurs in over $90 \%$ of NMS cases. In the present study, the detailed temporal changes in serum $\mathrm{CK}$ and degree of muscle rigidity, and the relationship between $\mathrm{CK}$ concentration and degree of muscle rigidity over the time course from fever onset, were evaluated in 24 affected patients. The results showed that serum CK peaked on day 2 after onset of fever and returned to within normal limits at day 12 . Mild muscle rigidity was observed before the onset of fever in 17 of 24 cases (71\%). Muscle rigidity was gradually exacerbated and worsened until day 4 after onset of fever. These findings confirm physicians' empirical understanding of serum CK concentrations and muscle rigidity in NMS based on data accumulated from numerous patients with the syndrome, and they indicate that serum CK may contribute to the early detection of NMS.
\end{abstract}

Keywords: neuroleptic malignant syndrome, creatine kinase, muscle rigidity

\section{Introduction}

Neuroleptic malignant syndrome (NMS) is a dangerous adverse response to antipsychotic drugs. The four major clinical symptoms of NMS are hyperthermia, severe muscle rigidity, autonomic dysfunction, and altered mental status. To date, several diagnostic criteria have been proposed for NMS. According to the diagnostic criteria of the fourth edition of the Diagnostic and Statistical Manual of Mental Disorders, Text Revision (DSM-IV-TR), ${ }^{1}$ the major symptoms are hyperthermia and severe muscle rigidity, and minor symptoms are diaphoresis, dysphagia, tremor, incontinence, changes in level of consciousness (ranging from confusion to coma), mutism, tachycardia, elevated or labile blood pressure, leucocytosis, and laboratory evidence of muscle injury (eg, elevated creatine kinase $[\mathrm{CK}]$ ). The presence of two or more of the ten minor symptoms is necessary for a diagnosis of NMS.

Levenson $^{2}$ suggested that elevated serum CK level is a major symptom used to diagnose NMS. This is in agreement with findings that serum CK is important for detecting NMS. ${ }^{3,4}$ On the other hand, it has been reported ${ }^{5-7}$ that elevated CK levels are of relatively little importance in the diagnosis of NMS. However, elevated CK concentrations have been found in over $90 \%$ of patients with NMS., ${ }^{2,5}$ As elevated $\mathrm{CK}$ is prevalent in the early stage of $\mathrm{NMS},{ }^{9}$ clarification of the temporal appearance of serum CK may assist in the diagnosis of NMS. 
Muscle rigidity is a major symptom used to diagnose NMS. Numerous case reports on NMS have been published, but there have been no detailed studies on how muscle rigidity develops during NMS. Furthermore, the relationship between CK concentration and the degree of muscle rigidity over the time course of NMS from onset has not been established.

In the present study, changes in serum $\mathrm{CK}$ and degree of muscle rigidity, and the temporal relationship between these factors, were evaluated in a large number of patients with NMS.

\section{Methods}

\section{Patients}

Between 1985 and 2012, 38 patients were treated in the authors' department for typical NMS, characterized by a fever (temperature $>38^{\circ} \mathrm{C}$ ), muscle rigidity, altered consciousness, autonomic dysfunction, and abnormal laboratory findings including elevated CK and leucocytosis. In a case of suspected NMS, body temperature and degree of muscle rigidity can be evaluated on a daily basis, but serum CK level cannot always be measured this regularly. In order to evaluate temporal alterations in serum CK, measurements must be taken several times during the episode of NMS. Serum CK levels could be evaluated only two or three times during the NMS episode in 12 of the 38 patients; therefore, it was difficult to evaluate temporal changes in serum CK in these 12 patients because the number of CK measurements was small. There were two patients in whom serum CK level could be evaluated first on day 7 after the onset of fever; therefore, it was difficult to evaluate the first weekly changes of serum CK in these two patients. Thus, these 14 patients were excluded from the present study. Overall, 24 patients in whom serum CK level could be evaluated temporally were selected for the present study. As already noted, all 24 patients had hyperthermia (temperature $>38^{\circ} \mathrm{C}$ ), muscle rigidity, altered consciousness, and autonomic disturbances including diaphoresis, tachycardia, labile blood pressure, and hypersalivation, and all cases met the criteria for NMS proposed in Levenson, ${ }^{2}$ Caroff and Mann, ${ }^{5}$ and the DSM$I V-T R .{ }^{1}$ A summary of patient characteristics is shown in Table 1 . We have previously reported monoamine levels in cerebrospinal fluid in patients with NMS. ${ }^{10-12}$ Specifically, cases $1-3$ in Table 1 correspond to cases 2, 4, and 5, respectively, in the first of these reports; cases 4-9 correspond to cases $1-4,6$, and 11 in the second report; and cases 10-15 correspond to cases 1-6 in the third report. Cases 16-23 have not been previously reported. Cases 1 and 24 have been reported recently. ${ }^{13}$
The summary of temporal changes in CK and muscle rigidity in individual patients is shown in Table 2 . The first day on which fever of unknown etiology is observed is considered day 1 . It should be noted that extrapyramidal symptoms persist in some patients with NMS after hyperthermia has subsided. ${ }^{14}$ Therefore, when patients become afebrile, disturbance of consciousness improves, and no autonomic dysfunction (ie, diaphoresis, tachycardia, and labile blood pressure) is observed, NMS is considered to have resolved. Taking case 1 as an example, NMS was sustained for 19 days. During the episode of NMS, serum CK was measured first on day 2, and six times thereafter. Including a further measurement after improvement of NMS, serum CK was measured a total of nine times. CK concentration peaked (722 IU/L) on day 3 . Case 1 did not present with muscle rigidity until day 8 after the onset of fever.

The NMS evaluation scale proposed by Sachdev ${ }^{15}$ was used to monitor temporal changes in muscle rigidity: 0 , nil (no rigidity); 1 , mild (slight rigidity present, particularly obvious on recruitment of muscles responsible for jaw clenching); 2, moderate (rigidity definitely present to a significant degree but produces no limitation of passive movement); and 3 , severe (rigidity that produces some limitation of passive movement). The NMS scale was proposed by Sachdev in 2005, enabling us to evaluate patients encountered since 2005 directly using this scale. Prior to 2005 , we evaluated the temporal degree of muscle rigidity in patients using the Ashworth Scale ${ }^{16}$ or the modified Ashworth Scale ${ }^{17}$ to evaluate the movement of joints. The obtained data enabled us to retrospectively evaluate the temporal degree by using the scale proposed by Sachdev.

Because we did not measure body temperature in the days before fever onset in all patients, we retroactively used temperature readings taken on day -3 and day -7 and added them to Table 3.

Temporal changes (mean \pm standard deviation) in body temperature, serum CK concentration, and degree of muscle rigidity during the course of NMS are shown in Table 3.

\section{Statistical analysis}

All statistical tests were conducted using SPSS version 11 for Windows (IBM Corporation, Armonk, NY, USA). A one-way analysis of variance with Dunnett's post hoc test was used to analyze changes in body temperature, serum CK levels, and degree of muscle rigidity. Baseline values of serum $\mathrm{CK}$ were considered to be those after recovery because values before fever were not available. 


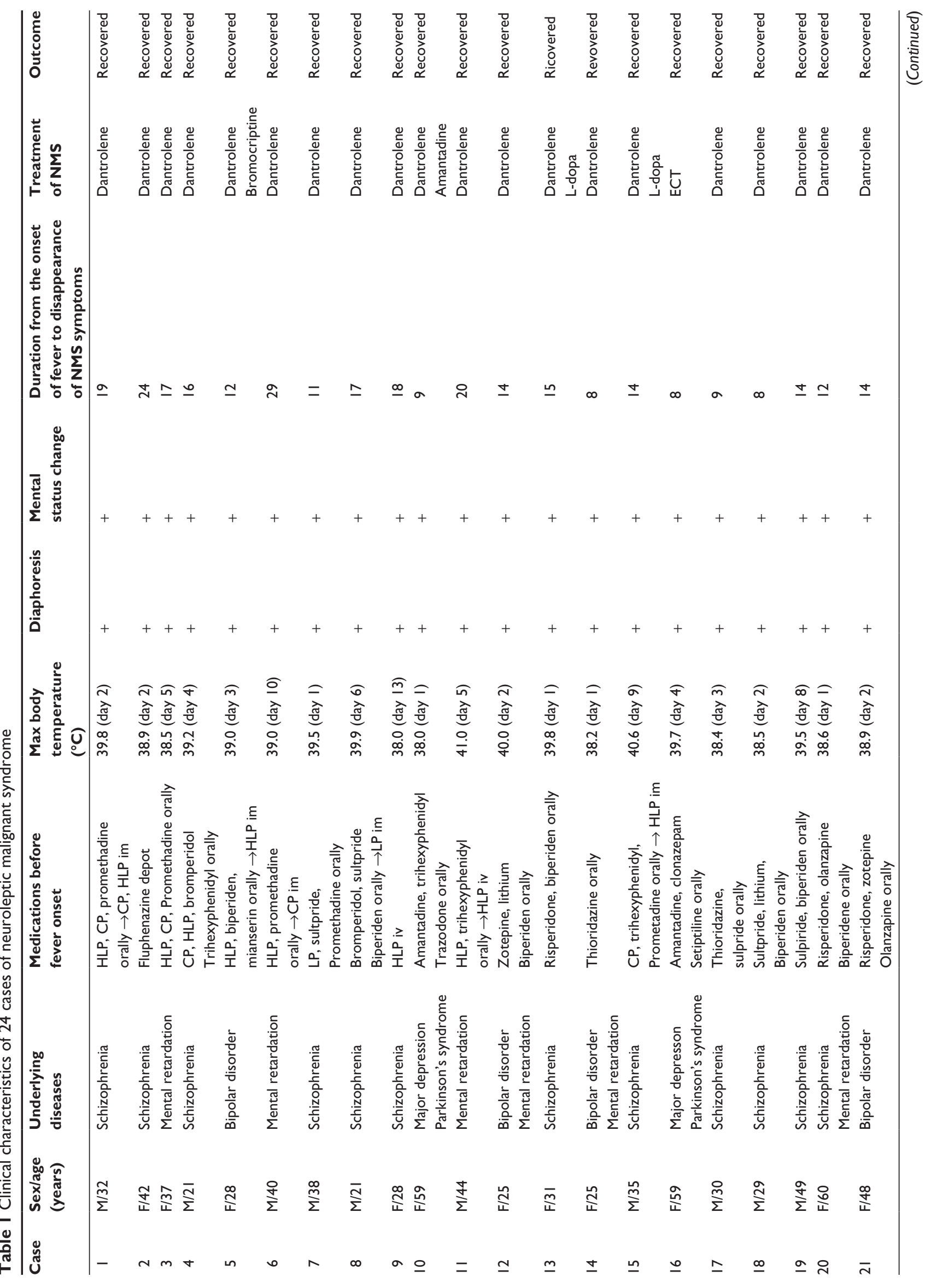




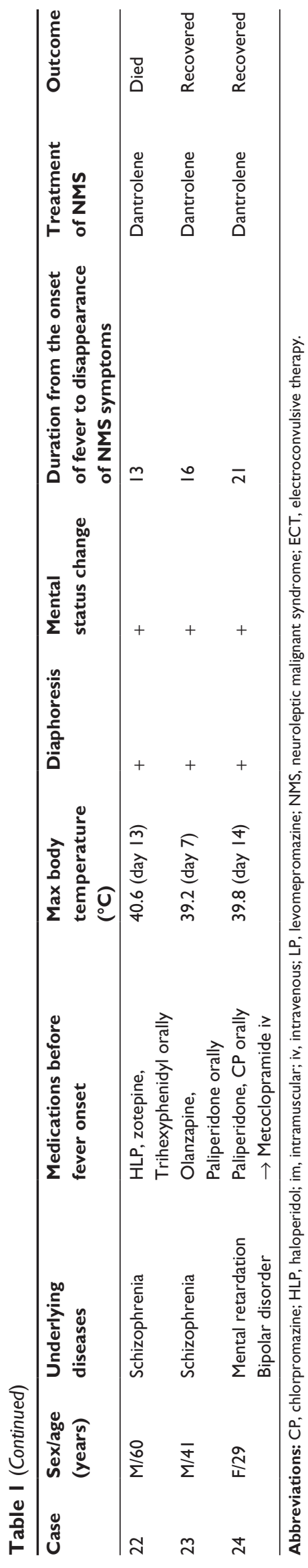

The relationship between serum CK and degree of muscle rigidity was examined using Pearson's correlation coefficient.

\section{Results}

The mean age of the study participants ( 12 men and 12 women) was 38 years (range 21-60 years). The mean highest temperature was $39.3^{\circ} \mathrm{C}$ (range $38.0^{\circ} \mathrm{C}-41.0^{\circ} \mathrm{C}$ ). The mean duration of NMS was 14.8 days (range 8-29 days). There was great variability among the peak $\mathrm{CK}$ concentrations, ranging from 495 to 44,880 IU/L. As shown in Table 1, there were only six patients who received intramuscular injections before fever onset. The mean number of CK measurements in each case was 7.4 (range 4-13) and the total number of measurements was 177 for the 24 cases. As also shown in Table 2, the most common day for CK concentration to peak was day 2 of NMS (seven cases), followed by days 3, 4 and 5 (three cases each). Mild muscle rigidity was observed in 17 of 24 cases ( $71 \%$ ) before the onset of fever. During the course of NMS, the strongest grade of rigidity, 3 , was seen in 20 patients and grade 2 was seen in four patients. NMS resolved in 23 patients without sequelae and one patient (case 22) died.

As shown in Table 3, overall serum CK peaked on day 2 and normalized to $165 \mathrm{IU} / \mathrm{L}$ (mean) (normal range 46-210) on day 12 . The degree of muscle rigidity peaked on day 4 and gradually decreased thereafter. Average body temperature peaked on days 1 and 2 and gradually decreased during NMS. Pearson's correlation test demonstrated that serum CK concentration was positively correlated with the degree of muscle rigidity $\left(\mathrm{R}^{2}=0.0392, P<0.01\right.$ [Figure 1]).

\section{Discussion}

The main findings of this study are: (1) serum CK peaked on day 2 after the onset of fever and returned to within normal limits by day 12; and (2) mild muscle rigidity was observed before the onset of fever in 17 of 24 cases (71\%) and was worst on day 4.

Serum CK is usually elevated following intramuscular injection, strenuous exercise, trauma, muscular injuries, acute psychosis, and restraint. Hence, elevated CK is not specific to NMS and is therefore not a reliable diagnostic parameter. The diagnosis of NMS should be based on four clinical symptoms: fever, altered consciousness, muscle rigidity, and autonomic dysfunction. However, it has been established that elevated CK is found in over $90 \%$ of patients with NMS. ${ }^{2,5,8}$ Therefore, if patients treated with antipsychotics develop a fever of unknown cause and their serum CK levels are elevated, NMS should be suspected in addition to other conditions in which 
Table 2 Creatine kinase and degree of muscle rigidity in 24 cases of neuroleptic malignant syndrome

\begin{tabular}{|c|c|c|c|c|c|c|c|}
\hline \multirow[t]{2}{*}{ Case } & \multicolumn{4}{|c|}{ Serum CK (IU/L) } & \multicolumn{3}{|l|}{ Muscle rigidity } \\
\hline & $\begin{array}{l}\text { Max } \\
\text { value }\end{array}$ & $\begin{array}{l}\text { The first day } \\
\text { of increased } \\
\text { CK (day) }\end{array}$ & $\begin{array}{l}\text { Peak day of } \\
\text { CK (day) }\end{array}$ & $\begin{array}{l}\text { Number of } \\
\text { measurement } \\
\text { of CK (times) }\end{array}$ & $\begin{array}{l}\text { Existence before } \\
\text { fever onset } \\
\text { (evaluation) }\end{array}$ & $\begin{array}{l}\text { Appearance day } \\
\text { (day) }\end{array}$ & $\begin{array}{l}\text { Peak } \\
\text { evaluation }\end{array}$ \\
\hline I & 722 & 2 & 3 & 9 & $-/(0)$ & 8 & 3 \\
\hline 2 & 1277 & 2 & 2 & 12 & $-/(0)$ & 4 & 3 \\
\hline 3 & 1236 & 4 & 8 & 8 & $+/(1)$ & Before onset of fever & 3 \\
\hline 4 & 2890 & 1 & 6 & 13 & $+/(1)$ & Before onset of fever & 3 \\
\hline 5 & 3854 & 3 & 3 & 6 & $+/(I)$ & Before onset of fever & 3 \\
\hline 6 & 495 & 2 & 2 & 8 & $-/(0)$ & 3 & 3 \\
\hline 7 & 3180 & 2 & 2 & 7 & $+/(1)$ & Before onset of fever & 2 \\
\hline 8 & 10,190 & 1 & 6 & 6 & $+/(I)$ & Before onset of fever & 3 \\
\hline 9 & 887 & 5 & 5 & 6 & $-/(0)$ & 5 & 2 \\
\hline 10 & 9580 & I & 1 & 8 & $+/(I)$ & Before onset of fever & 3 \\
\hline II & 1688 & 1 & 8 & 8 & $+/(1)$ & Before onset of fever & 3 \\
\hline 12 & 44,880 & 2 & 2 & 7 & $+/(1)$ & Before onset of fever & 3 \\
\hline 13 & 22423 & I & 2 & 9 & $+/(1)$ & Before onset of fever & 3 \\
\hline 14 & 1558 & 3 & 4 & 5 & $-/(0)$ & 2 & 2 \\
\hline 15 & 2238 & I & 10 & 6 & $+/(1)$ & Before onset of fever & 3 \\
\hline 16 & 777 & 5 & 5 & 7 & $+/(2)$ & Before onset of fever & 3 \\
\hline 17 & 7346 & 3 & 3 & 7 & $+/(1)$ & Before onset of fever & 3 \\
\hline 18 & 772 & 2 & 2 & 6 & $+/(1)$ & Before onset of fever & 3 \\
\hline 19 & 9610 & 4 & 9 & 5 & $-/(0)$ & 7 & 2 \\
\hline 20 & 1426 & I & 5 & 8 & $+/(2)$ & Before onset of fever & 3 \\
\hline 21 & 3949 & 1 & 2 & 5 & $+/(1)$ & Before onset of fever & 3 \\
\hline 22 & 839 & 4 & 4 & 4 & $+/(1)$ & Before onset of fever & 3 \\
\hline 23 & 2755 & I & 7 & 11 & $+/(1)$ & Before onset of fever & 3 \\
\hline 24 & 4273 & 4 & 4 & 6 & $-/(0)$ & 12 & 3 \\
\hline
\end{tabular}

Note: The degree of muscle rigidity was evaluated using the NMS scale proposed by Sachdev. ${ }^{15}$

Abbreviations: CK, creatine kinase; NMS, neuroleptic malignant syndrome.

Table 3 Temporal changes in body temperature, serum creatine kinase, and muscle rigidity in 24 cases of neuroleptic malignant syndrome

\begin{tabular}{|c|c|c|c|}
\hline & Body temperature & Serum CK & $\begin{array}{l}\text { Evaluation of } \\
\text { muscle rigidity }\end{array}$ \\
\hline Before fever onset & $36.6 \pm 0.5(n=20)$ & - & Day $-10.78 \pm 0.58$ \\
\hline (Day -3 and Day -7 ) & & & $(n=24)$ \\
\hline Day I & $38.2 \pm 0.7 *(n=24)$ & $1414 \pm 2675(n=11)$ & $1.00 \pm 0.88(n=24)$ \\
\hline Day 2 & $38.2 \pm 0.7^{*}(n=24)$ & $7853 \pm 13,256$ ** $(n=11)$ & $1.39 \pm 1.05(n=24)$ \\
\hline Day 3 & $38.1 \pm 0.9 *(n=24)$ & $4773 \pm 627 \mid(n=13)$ & $1.65 \pm 1.12^{*}(n=24)$ \\
\hline Day 4 & $38.1 \pm 0.8^{*}(n=24)$ & $1795 \pm 2715(n=14)$ & $1.91 \pm 1.05^{*}(n=24)$ \\
\hline Day 5 & $37.8 \pm 0.9 *(n=23)$ & $1191 \pm 1895(n=12)$ & $1.78 \pm 0.97 *(n=24)$ \\
\hline Day 6 & $37.7 \pm 0.7 *(n=24)$ & $2023 \pm 2798(n=11)$ & $1.69 \pm 1.08 *(n=24)$ \\
\hline Day 7 & $37.5 \pm 0.6 *(n=23)$ & $938 \pm 1106(n=11)$ & $1.56 \pm 1.05 *(n=24)$ \\
\hline Day 8 & $37.7 \pm 0.7^{*}(n=23)$ & $1502 \pm 1977(n=13)$ & $1.52 \pm 0.92 *(n=24)$ \\
\hline Day 9 & $37.7 \pm 0.9 *(n=24)$ & $959 \pm 2411(n=14)$ & $1.17 \pm 0.91 *(n=24)$ \\
\hline Day 10 & $37.6 \pm 0.8^{*}(n=23)$ & $693 \pm 788(n=5)$ & $1.08 \pm 0.92(n=24)$ \\
\hline Day II & $37.5 \pm 0.8^{*}(n=2 I)$ & $380 \pm 446(n=10)$ & $1.04 \pm 1.04(n=24)$ \\
\hline Day 12 & $37.5 \pm 0.9 *(n=21)$ & $165 \pm 92(n=9)$ & $0.78 \pm 1.01 \quad(n=24)$ \\
\hline- & - & - & - \\
\hline After recovery & $36.8 \pm 0.3(n=23)$ & $104 \pm 7 \mid(n=23)$ & $0.14 \pm 0.46(n=23)$ \\
\hline
\end{tabular}

Notes: Data are expressed as mean \pm SD values. Data are shown until day 12 from the onset of fever because mean serum creatine kinase was normalized on day 12 (normal range 46-2/0 IU/L). Statistically significant differences are indicated as follows: $* P<0.05$ (versus before fever onset); $* * P=0.007$ (versus after recovery). Abbreviations: $\mathrm{CK}$, creatine kinase; SD, standard deviation. 
IU/L

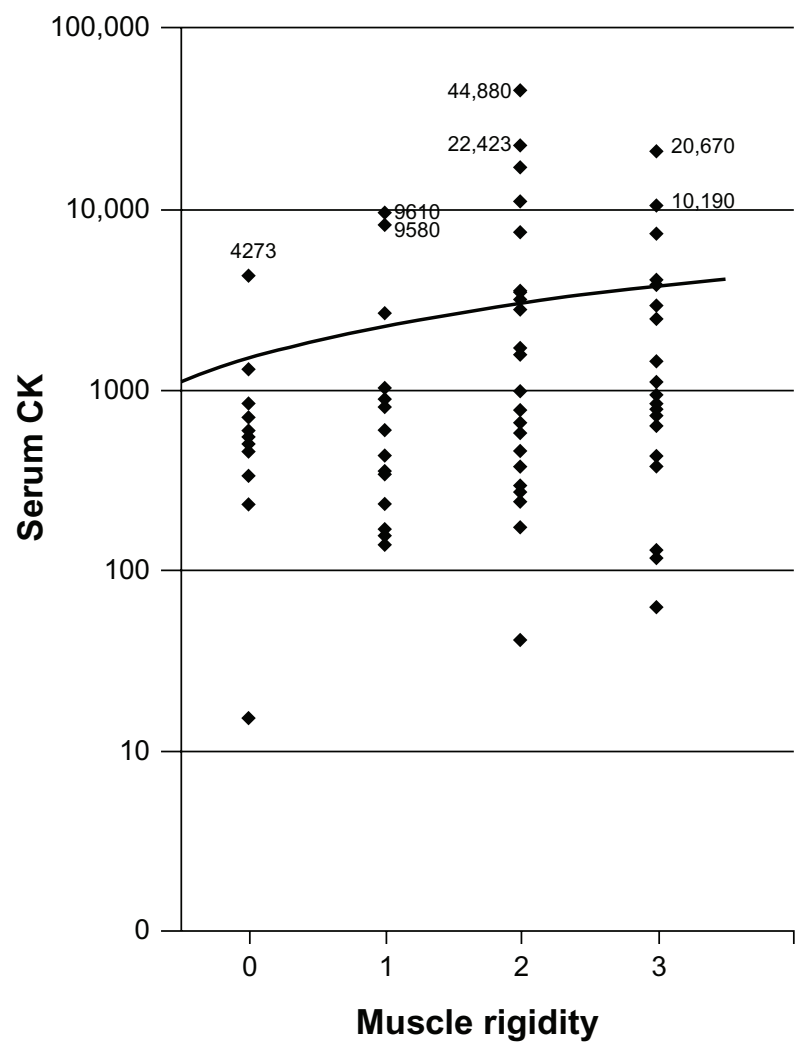

Figure I Correlation between creatine kinase concentration and degree of muscle rigidity in 24 cases of neuroleptic malignant syndrome.

Notes: Pearson's $R^{2}=0.0392 ; P<0.01 ; \mathrm{n}=177$.

Abbreviation: $C K$, creatine kinase.

serum CK levels are elevated. If muscle rigidity is observed, the patient has a higher risk of developing NMS. Therefore, it is important for diagnosing NMS to understand how serum $\mathrm{CK}$ concentration and the degree of muscle rigidity change during the course of NMS.

Harsch $^{18}$ reported that CK concentration peaked 24-48 hours after onset in nine cases of NMS, and Rosebush and Stewart ${ }^{19}$ reported among 24 episodes of NMS a peak on days 2 and 3 after onset in $64 \%$ of episodes, on days 4 to 7 in $29 \%$, and on day 1 in $7 \%$. The present study is the first to examine temporal changes in serum CK in numerous NMS cases, and the present results confirm the above findings.

Muscle rigidity was observed in 17 of 24 cases (71\%) before the onset of fever. Velamoor et $\mathrm{al}^{20}$ investigated 222 NMS patients previously reported and concluded that mental status change or muscle rigidity was the initial manifestation in $82.3 \%$ of cases. The present research findings support their results to some extent. Velamoor et al did not evaluate when the degree of muscle rigidity became most severe; however, the results of the present study demonstrate a peak on day 4 after the onset of fever. Taken together, in many cases it appears that serum CK concentration rapidly increases after the onset of fever, peaks on day 2 , and normalizes on day 12 . By contrast, mild muscle rigidity is present at the onset of NMS in $71 \%$ of cases and peaks on day 4. On the other hand, we reported exceptional cases of NMS with regard to temporal changes in serum $\mathrm{CK}$ and muscle rigidity. ${ }^{13}$ As muscle rigidity first appeared after serum CK had normalized in cases 1 and 24, physicians should be aware of the existence of these uncommon findings.

We calculated Pearson's correlation coefficient to assess the relationship between serum CK levels and degree of muscle rigidity and noted a significant positive but weak correlation $(r=0.198)$. Serum CK is elevated in numerous situations. To conclude that elevated CK is significantly associated with degree of muscle rigidity, it is necessary to rule out other factors including intramuscular injection, strenuous exercise, and acute catatonia. Therefore, at present, we are unable to ascertain whether elevated CK is associated with muscle rigidity.

Average body temperature peaked on days 1 and 2, and gradually decreased during the course of NMS in 24 cases. As shown in Table 1, the day of maximal body temperature was variable. It is possible that low-grade fever preceded the appearance of hyperthermia (temperature $>38^{\circ} \mathrm{C}$ ). As we did not measure body temperature on the day prior to hyperthermia onset in any cases, it is unclear whether body temperature generally peaks on day 1 or 2 of NMS.

Some limitations of the present study should be considered. First, the study was carried out retrospectively to assess the degree of muscle rigidity. It will be necessary to examine this condition prospectively in a large sample population with NMS. Second, serum CK was randomly measured in each patient; daily measurement of CK is difficult because NMS is rare and measurement of serum $\mathrm{CK}$ is not a routine examination.

In conclusion, serum CK levels peaked on the second day after onset of fever. Mild muscle rigidity was observed in about $71 \%$ of 24 cases, and the degree of muscle rigidity was worst on the fourth day, after which it gradually resolved. The findings of our study confirm physicians' empirical knowledge of serum CK concentration and muscle rigidity in NMS based on the data accumulated from numerous patients with this syndrome. 


\section{Acknowledgment}

The authors gratefully acknowledge Dr Koichirou Kishi, Professor of Jichi Medical University Center for Information, for his support with the statistical analysis.

\section{Disclosure}

The authors report no conflicts of interest in this work.

\section{References}

1. American Psychiatric Association. Medication-induced movement disorders: neuroleptic malignant syndrome. In: Diagnostic and Statistical Manual of Mental Disorders: DSM-IV-TR, 4th ed. American Psychiatric Association: Washington, DC; 2000:795-798.

2. Levenson JL. Neuroleptic malignant syndrome. Am J Psychiatry. 1985;142(10):1137-1145.

3. Carroll BT, Surber SA. The problem of atypical neuroleptic malignant syndrome: a case report. Psychiatry (Edgmont). 2009;6(7):45-47.

4. Neuhut R, Lindenmayer JP, Silva R. Neuroleptic malignant syndrome in children and adolescents on atypical antipsychotic medication: a review. J Child Adolescent Psychopharmacology. 2009;19(4):415-422.

5. Caroff SN, Mann SC. Neuroleptic malignant syndrome. Med Clin North Am. 1993;77(1):185-202.

6. Buckley PF, Hutchinson M. Neuroleptic malignant syndrome. J Neurol Neurosur Psychiatry. 1995;58(3):271-273.

7. Susman VL. Clinical management of neuroleptic malignant syndrome. Psychiatr Q. 2001;72(4):325-336.

8. Addonizio G, Susman VL, Roth SD. Neuroleptic malignant syndrome: review and analysis of 115 cases. Biol Psychiatry. 1987;22(8): 1004-1020.
9. Védie C, Poinso F, Hemmi F, Rivet B. Major symptoms and differential diagnosis of neuroleptic malignant syndrome: three case reports. Eur Psychiatry. 2000;15(5):334-337.

10. Nisijima K, Ishiguro T. Neuroleptic malignant syndrome: a study of CSF monoamine metabolism. Biol Psychiatry. 1990;27(3):280-288.

11. Nisijima K, Ishiguro T. Cerebrospinal fluid levels of monoamine metabolites and gamma-aminobutyric acid in neuroleptic malignant syndrome. J Psychiat Res. 1995;29(3):233-244.

12. Nisijima K, Shioda K, Iwamura T. Neuroleptic malignant syndrome and serotonin syndrome. Prog Brain Res. 2007;162:81-104.

13. Nisijima K. Elevated creatine kinase does not necessarily correspond temporally with onset of muscle rigidity in neuroleptic malignant syndrome: a report of two cases. Neuropsychiatr Dis Treat. 2012;8: 615-618.

14. Panagariya A, Sharma B, Singh R, Agarwal V, Dev A. The neuroleptic malignant syndrome: a report of 14 cases from north India. Neurol India. 2007;55(2):166-168.

15. Sachdev PS. A rating scale for neuroleptic malignant syndrome. Psychiatry Res. 2005;135(3):249-256.

16. Ashworth B. Preliminary trial of carisoprodol in multiple sclerosis Practitioner. 1964;192:540-542.

17. Bohannon RW, Smith MB. Interrater reliability of a modified Ashwworth scale of muscle spasticity. Phys Ther. 1987;67(2):206-207.

18. Harsch HH. Neuroleptic malignant syndrome: physiological and laboratory findings in a series of nine cases. J Clin Psychiatry. 1987; 48(8):328-333.

19. Rosebush P, Stewart T. A prospective analysis of 24 episodes of neuroleptic malignant syndrome. Am J Psychiatry. 1989;146(6): $717-725$.

20. Velamoor VR, Norman RM, Caroff SN, Mann SC, Sullivan KA, Antelo RE. Progression of symptoms in neuroleptic malignant syndrome. J Nerv Ment Dis. 1994;182(3):168-173.
Neuropsychiatric Disease and Treatment

\section{Publish your work in this journal}

Neuropsychiatric Disease and Treatment is an international, peerreviewed journal of clinical therapeutics and pharmacology focusing on concise rapid reporting of clinical or pre-clinical studies on a range of neuropsychiatric and neurological disorders. This journal is indexed on PubMed Central, the 'PsycINFO' database and CAS.

\section{Dovepress}

The manuscript management system is completely online and includes a very quick and fair peer-review system, which is all easy to use. Visit http://www.dovepress.com/testimonials.php to read real quotes from published authors. 